\title{
Nonuniform cosmological birefringence and active galactic nuclei
}

\begin{abstract}
Marc Kamionkowski
California Institute of Technology, Mail Code 350-17, Pasadena, California 91125, USA

(Received 21 April 2010; revised manuscript received 28 April 2010; published 25 August 2010)

The CMB constrains the cosmological-birefringence rotation angle to be $|\alpha| \lesssim 1^{\circ}(1 \sigma)$ out to redshifts $z \simeq 1100$ for a rotation that is uniform across the sky. However, the rotation angle $\alpha(\theta, \phi)$ may vary with position $(\theta, \phi)$ on the sky, and if so, then it can be sought in current and future active galactic nuclei data. An upper limit $\left\langle\alpha^{2}\right\rangle^{1 / 2} \lesssim 3.7^{\circ}$ to the scatter in the position-angle-polarization offsets in a sample of only $N=9$ active galactic nuclei already constrains the rotation spherical-harmonic coefficients to $(4 \pi)^{-1 / 2} \alpha_{l m} \lesssim 3.7^{\circ}$ and constrains the power spectrum for $\alpha$ in models where it is a stochastic field. Future constraints can be improved with more sources and by analyzing well-mapped sources with a tensor-harmonic decomposition of the polarization.
\end{abstract}

DOI: $10.1103 /$ PhysRevD.82.047302

PACS numbers: $98.80 . E s, 98.54 . \mathrm{Cm}$

Introduction.-There is a very active quest to understand dark energy [1], and quintessence models [2] provide a promising set of effective theories. A pseudo-NambuGoldstone field provides an attractive quintessence candidate, and such a field should have a coupling to the ChernSimons term of electromagnetism [3]. This coupling gives rise to cosmological birefringence (CB), a frequencyindependent rotation by some angle $\alpha$, of the linear polarization of photons as they propagate over cosmological distances [3,4]. In the simplest models, the rotation angle $\alpha$ is uniform across the sky, in which case $\mathrm{CB}$ gives rise to parity-violating $T B$ and $E B$ correlations in CMB maps [5]. Null searches for such correlations now constrain the rotation over the redshift range $0<z \lesssim 1100$ to be $\alpha=$ $-0.25^{\circ} \pm 0.58^{\circ}[6]$.

However, several recent papers have introduced quintessence models in which the rotation angle $\alpha(\theta, \phi)$ varies as a function of position $(\theta, \phi)$ on the sky [7], and similar phenomena may arise in some dark-matter models [8]. References [9] have described how to measure this rotation angle, as a function of position on the sky, with the CMB, but the algorithm has not yet been applied to data. The Wilkinson Microwave Anisotropy Probe satellite should be sensitive (at $1 \sigma$ ) to spherical-harmonic coefficients of the rotation as small as $(4 \pi)^{-1 / 2}\left|\alpha_{l m}\right| \sim 2.3^{\circ}$, for $l \lesssim 400$, and the recently launched Planck satellite should reach $(4 \pi)^{-1 / 2}\left|\alpha_{l m}\right| \sim 0.07^{\circ}$ for $l \lesssim 800$ [9].

Radio [4,10-14] and UV [15,16] data on active galactic nuclei (AGN) can also be used to search for CB. AGN are often elongated and polarized. While AGN may be complicated objects, symmetry considerations suggest that on average the mean polarization should be aligned or perpendicular to the position angle of the source. CB would, by rotating the polarization, give rise to a nonzero mean offset between the position angles and polarizations measured in a large number of sources [4,10,11]. Likewise, if a detailed map of the intensity and polarization of an individual source can be made $[12,13,15]$, then, on average, the intensity gradients and polarization within that source should be aligned or perpendicular, if there is no CB. This more detailed analysis may allow competitive, or even stronger, constraints on $\alpha$ from a smaller number of sources.

One precisely imaged radio source (3C 9) at a redshift $z \simeq 2$ constrains $\alpha=2^{\circ} \pm 3^{\circ}$ out to this distance [13,17]. A stronger bound, $\alpha=-0.6^{\circ} \pm 1.5^{\circ}$, can be obtained from a larger number of well-mapped radio sources, but only at smaller redshifts [3]. A recent UV sample [16] of eight AGN at redshifts $z \gtrsim 2$ constrains $\alpha=-0.7^{\circ} \pm$ $2.0^{\circ}$. In this paper, I show that AGN can be used to constrain the multipole moments $\alpha_{l m}$ for a nonuniform rotation.

There was a brief flurry in the 1990s of searches for a rotation with a dipole dependence on position [11-14], following a claimed detection [10]. Here I revisit and update such measurements and generalize to higher- $l$ moments. I search the recent UV data [16], combined with the radio constraint from 3C 9 [13], and find no evidence for any rotation with a dipole or quadrupole dependence on position. I constrain the $\alpha_{l m}$ (for any $l$ ) to $(4 \pi)^{-1 / 2} \alpha_{l m} \lesssim$ $3.7^{\circ}(1 \sigma)$, and I place a constraint on the power spectrum for $\alpha$ for theories that predict that $\alpha(\theta, \phi)$ is a stochastic field. As a preface, I discuss the derivation of the constraint, $\alpha=-0.7^{\circ} \pm 2.0^{\circ}$ (to redshifts $z \simeq 2$ ), from the $\mathrm{UV}$ data, a result that is strengthened to $\alpha=-0.1^{\circ} \pm 1.7^{\circ}$ if the radio data on $3 \mathrm{C} 9$ is included. Finally, I discuss how the analysis of high-resolution intensity-polarization maps of individual sources can be optimized, using tensorharmonic techniques similar to those for CMB polarization and weak gravitational lensing.

Prelude: A uniform rotation.-Table I reproduces data on eight UV sources from Ref. [16] as well as radio data on 3C 9 [13,17]. Listed there are the positions $\left(\theta_{i}, \phi_{i}\right)$, position-angle-polarization offsets $\alpha_{i}$, and measurement errors $\sigma_{i}$ on these offsets.

Let us first test with this data whether there is a rotation, by an angle $\alpha$, that is uniform across the sky. We will also determine the scatter $\sigma_{p}$ in the measurements of $\alpha$. To 
estimate the mean offset from the data, we use the minimum-variance estimator, $\hat{\alpha}=\left[\sum_{i} \alpha_{i} \sigma_{i}^{-2}\right]\left[\sum_{i} \sigma_{i}^{-2}\right]^{-1}$. The error to our measurement of $\alpha$ is then the inverse root of the denominator. We find for the UV data in Table I $\alpha=$ $-0.7 \pm 2.0$. Including the radio source $3 \mathrm{C} 9$ improves the minimum-variance constraint on $\alpha=-0.1^{\circ} \pm 1.7^{\circ}$. In general, it could be that the sample contains a significant intrinsic scatter in the offsets, in which case the minimumvariance error would underestimate the true error in $\alpha$. For example, one extremely well-measured and statistically significant nonzero offset might suggest nonzero $\mathrm{CB}$, but could alternatively be due to an intrinsic offset in the source. Still, the measured offsets for the current sample are well within their measurement errors, and so the error obtained here is probably sound. The error $\sigma_{\alpha}^{2}=\sum_{i}\left(\alpha_{i}-\right.$ $\hat{\alpha})^{2} /[N(N-1)]$ obtained from the measured dispersion is in fact a bit smaller, $\sigma_{\alpha}=1.4^{\circ}$, suggesting that the reported measurement errors in this sample may be a bit high and the true constraint a bit stronger.

The scatter $\sigma_{p}$ in the offsets is determined from the data via

$$
\sigma_{p}^{2}=\left[\sum_{i}\left(\alpha_{i}-\hat{\alpha}\right)^{2} \sigma_{i}^{-2}\right]\left[\sum_{i} \sigma_{i}^{-2}-\sum_{i} \sigma_{i}^{-4} / \sum_{i} \sigma_{i}^{-2}\right]^{-1}
$$

The nine sources in Table I result in $\sigma_{p}=2.9^{\circ}$, a result that will be used below. Note that the weighted estimate of the scatter in Eq. (1) is a bit smaller than the value $4.4^{\circ}$ obtained if an unweighted estimator, $\sigma_{p}^{2}=(N-$ $1)^{-1} \sum_{i}\left(\alpha_{i}-\hat{\alpha}\right)^{2}$, for the variance is used, an indication that the unweighted variance in this data is due primarily to measurement error, not intrinsic scatter.

Nonuniform rotation.-Now suppose we wish to test if there is a single $l m$ spherical-harmonic variation in $\alpha(\theta, \phi)$, i.e., that $\alpha(\theta, \phi)=\alpha_{l m} Y_{l m}(\theta, \phi)$, for some given $l$ and $m$. Then each data point would provide an estimator $\hat{\alpha}_{l m}^{i}=\left(\alpha_{i}-\hat{\alpha}\right) / Y_{l m}\left(\theta_{i}, \phi_{i}\right)$, with variance $\left\langle\left|\hat{\alpha}_{l m}^{i}\right|^{2}\right\rangle=$ $\sigma_{i}^{2} /\left|Y_{l m}\left(\theta_{i}, \phi_{i}\right)\right|^{2}$. Note that $\hat{\alpha}_{l m}^{i}$ is complex, and the variances to the real and imaginary parts are each $\left\langle\left|\hat{\alpha}_{l m}^{i}\right|^{2}\right\rangle / 2$.

TABLE I. The $\theta_{i}-\phi_{i}$ coordinates, offsets $\alpha_{i}$, and measurement errors $\sigma_{i}$ for the eight sources listed in Ref. [16] plus the (last) radio source 3C 9 (from Ref. [13]).

\begin{tabular}{lcccc}
\hline \hline $\mathrm{i}$ & $\theta_{i}(\mathrm{deg})$ & $\phi_{i}(\mathrm{deg})$ & $\alpha_{i}(\mathrm{deg})$ & $\sigma_{i}(\mathrm{deg})$ \\
\hline 1 & 78 & 34 & -1.0 & 3.5 \\
2 & 66 & 146 & -0.3 & 4.4 \\
3 & 109 & 128 & 1.6 & 4.5 \\
4 & 90 & 213 & -8.0 & 8.0 \\
5 & 93 & 191 & -4.0 & 8.8 \\
6 & 68 & 307 & -4.0 & 9.0 \\
7 & 114 & 317 & 4.6 & 9.7 \\
8 & 103 & 20 & 5.0 & 16 \\
9 & 5 & 105 & 2 & 3 \\
\hline \hline
\end{tabular}

The minimum-variance estimator $\hat{\alpha}_{l m}$ obtained from all $N$ data points is obtained by adding all the $N$ individual $\hat{\alpha}_{l m}^{i}$ estimators with inverse-variance weighting; i.e.,

$$
\hat{\alpha}_{l m}=\left[\sum_{i} \frac{\alpha_{i}-\hat{\alpha}}{\sigma_{i}^{2}} Y_{l m}^{*}\left(\theta_{i}, \phi_{i}\right)\right] /\left[\sum_{i} \frac{\left|Y_{l m}\left(\theta_{i}, \phi_{i}\right)\right|^{2}}{\sigma_{i}^{2}}\right],
$$

with variance given by the inverse of the denominator in this expression. The results of such an analysis of the nine sources in Table I are presented in Table II. There is no evidence for any nonzero $\alpha_{l m}$ for $l \leq 2$.

Strictly speaking, there will be correlations between the estimators for these lowest multipole moments due to the irregular/sparse sky coverage of these eight sources. By including the $\hat{\alpha}$ term in the estimator, I attribute any evidence for a signal — should any exist - to the monopole in case irregular sky coverage correlates the monopole and some other moment. This term should become irrelevant in the limit $N \rightarrow \infty$, with a population of sources spread uniformly throughout the sky. Given the illustrative nature of this analysis, I leave the full covariance matrix for the different moments for future work. For now, I simply caution that the derived upper limits may be weakened, perhaps by up to a factor $\sim \sqrt{2}$, by these correlations.

The values of the individual $\hat{\alpha}_{l m}$ 's, for a given $l$, depend on the choice of coordinate system. To test for a nonuniform $\mathrm{CB}$ in a rotationally invariant way, one must evaluate the rotational invariants $\hat{C}_{l}=\sum_{m=-l}^{l}\left|\hat{\alpha}_{l m}\right|^{2} /(2 l+1)$. Doing so, no evidence of a nonuniform $\mathrm{CB}$ is found for the dipole $(l=1)$ and quadrupole $(l=2)$. Rough upper limits to the dipole and quadrupole amplitudes can be obtained from the noise: $\sqrt{C_{1} /(4 \pi)} \lesssim 2.3^{\circ}$ and $\sqrt{C_{2} /(4 \pi)} \lesssim 1.9^{\circ}$.

Higher-l moments. - Since we have in the current analysis only eight data points, it is not really possible to measure any $\alpha_{l m}$ 's with $l \gtrsim 2$. However, if there were a nonzero $\alpha_{l m}$ for some high $l$, it would give rise to a scatter in the measured $\alpha_{i}$ 's with variance, $\left\langle\alpha^{2}\right\rangle=(4 \pi)^{-1} \times$ $\int d \hat{\boldsymbol{n}}[\alpha(\theta, \phi)]^{2}=\left|\alpha_{l m}^{2}\right| /(4 \pi)$. If the sources are randomly distributed on the sky at points with angular separations $\Delta \theta \gg \pi / l$, then this variance $\left\langle\alpha^{2}\right\rangle$ cannot be larger than the variance in the data. The variance measured from the data in Table I is roughly $\left(2.9^{\circ}\right)^{2}$, but there is a sample error

TABLE II. The measured $\alpha_{l m}$ obtained from the data in Table I.

\begin{tabular}{lccc}
\hline \hline$l$ & $m$ & $(4 \pi)^{-1 / 2} \hat{\alpha}_{l m}(\mathrm{deg})$ & $(4 \pi)^{-1 / 2}\left\langle\left|\alpha_{l m}\right|^{2}\right\rangle^{1 / 2}(\mathrm{deg})$ \\
\hline 1 & 0 & -2.9 & 3.4 \\
1 & 1 & $-0.7-0.3 i$ & 1.4 \\
2 & 0 & 0.2 & 2.0 \\
2 & 1 & $1.1+0.2 i$ & 2.3 \\
2 & 2 & $0.2-0.5 i$ & 1.3 \\
\hline \hline
\end{tabular}


to this variance, of roughly $\sqrt{2 / N}$, where $N$ is the number of (statistically significant) data points, which I estimate to be 5 . I therefore take, as a rough upper limit, $\left\langle\alpha^{2}\right\rangle^{1 / 2} \lesssim$ $3.7^{\circ}$, implying $\left|\alpha_{l m}\right|^{2} / 4 \pi \lesssim\left(3.7^{\circ}\right)^{2}$. The upper limit to any $C_{l}$ is similar: $C_{l} / 4 \pi \lesssim\left(3.7^{\circ}\right)^{2}$ for any individual higher $l$.

Stochastic values of $\alpha$.- Theories with a spatially varying $\alpha(\theta, \phi)$ generally predict that $\alpha(\theta, \phi)$ is a realization of a random field with some given power spectrum $C_{l}$. Such a theory results in a variance in the measured offsets of $\left\langle\alpha^{2}\right\rangle=\sum_{l}(2 l+1) C_{l} /(4 \pi)$ which, again, must be $\lesssim$ $\left(3.7^{\circ}\right)^{2}$. For example, suppose some theory predicts a scale-invariant spectrum, $l(l+1) C_{l}=2 C_{1} \exp \left(-l^{2} / l_{c}^{2}\right)$ from $l=1$ out to some cutoff moment $l_{c}$ with an amplitude parametrized by the dipole moment $C_{1}$. Then approximating for this model $\left\langle\alpha^{2}\right\rangle \simeq C_{1} \ln \left(7 l_{c}\right) / \pi$, we find the dipole to be constrained to $C_{1} / 4 \pi \lesssim(1 / 4)\left\langle\alpha^{2}\right\rangle / \ln \left(7 l_{c}\right) \simeq$ $\left(0.7^{\circ}\right)^{2} / \ln \left(l_{c} / 200\right)$. Note that the finite angular size $\delta$ of the images limits the effective $l_{c} \lesssim 200\left(\delta / 1^{\circ}\right)^{-1}$, even if the theory allows it to be much larger.

Of course, an upper limit to $\left\langle\alpha^{2}\right\rangle$ can be obtained [7] by the upper limit to the $B$-mode polarization induced by rotation. Roughly speaking, a fraction $\sim 2\left\langle\alpha^{2}\right\rangle^{1 / 2}$ of the primordial $E$ mode is converted to the $B$ mode, implying a $B$-mode power spectrum $C_{l}^{B B} \sim 4\left\langle\alpha^{2}\right\rangle^{1 / 2} C_{l}^{E E}$. Given current upper limits $C_{l}^{B B} \lesssim 0.1 C_{l}^{E E}$ [7], we infer a $B$-mode upper limit $\left\langle\alpha^{2}\right\rangle^{1 / 2} \lesssim 10^{\circ}$, weaker than the AGN bound derived here.

Stochastic values of $\alpha$.-Early measurements of $\mathrm{CB}$ $[4,10,11]$ considered simply the offset between the image position angle and the mean polarization averaged over the entire image. But this averaging erases much of the information available in the source $[12,13,15]$. If a highresolution map of the intensity and polarization of a given source is available, then the offset between the intensity gradient and the polarization throughout a given source can provide a far more precise measurement of the mean offset for that particular source. The sensitivity to CB from a handful of well-resolved sources can thus compete with that of hundreds of unresolved sources.

Still, one can do better in terms of measuring the offset $\alpha$ from a given well-resolved source than prior analyses by using techniques developed to quantify temperaturepolarization correlation functions in the CMB $[18,19]$, and also galaxy-shape correlations induced by weak gravitational lensing [20]. These techniques deal, for example, with the ambiguity in the direction of the linear polarization and also with optimizing low signal-to-noise measurements. They allow the full two-point intensity-polarization correlations to be used, rather than simply the correlations at zero lag (as prior analyses have used). They provide additional information on $\alpha$ from the polarization alone, even without cross correlation with the intensity, something that could not be done with the more heuristic intensity-gradient-polarization correlation. These tech- niques are analogous to those for measuring a uniform rotation angle with the CMB [5].

Suppose that we have a resolved map of the intensity $I\left(\theta_{x}, \theta_{y}\right)$ and Stokes parameters $Q\left(\theta_{x}, \theta_{y}\right)$ and $U\left(\theta_{x}, \theta_{y}\right)$ of a given radio source, where $\theta_{x}$ and $\theta_{y}$ are coordinates in the image plane. One first Fourier transforms, $\tilde{I}\left(l_{x}, l_{y}\right)=$ $\int d^{2} \theta e^{i \vec{l} \cdot \vec{\theta}} I(\vec{\theta})$, and similarly for $\tilde{Q}(\vec{l})$ and $\tilde{U}(\vec{l})$, from which are obtained the rotational invariants,

$$
\left(\begin{array}{c}
\tilde{E}_{\vec{l}} \\
\tilde{B}_{\vec{l}}
\end{array}\right)=\frac{1}{2\left(l_{x}^{2}+l_{y}^{2}\right)}\left(\begin{array}{cc}
\left(l_{x}^{2}-l_{y}^{2}\right) & 2 l_{x} l_{y} \\
2 l_{x} l_{y} & -\left(l_{x}^{2}-l_{y}^{2}\right)
\end{array}\right)\left(\begin{array}{c}
\tilde{Q}_{\vec{l}} \\
\tilde{U}_{\vec{l}}
\end{array}\right) .
$$

From these, the six power spectra $P_{l}^{X X^{\prime}}=\left\langle\tilde{X}(\vec{l}) \tilde{X}^{\prime}(\vec{l})^{*}\right\rangle$ (where the angle brackets denote an ensemble average) can be obtained, where $\left\{X, X^{\prime}\right\}=\{I, E, B\}$. The $B$ modes have opposite parity from the $I$ and $E$ modes, and so we should have $P_{l}^{I B}=P_{l}^{E B}=0$, if parity is preserved. While any given source may, in principle, have some handedness, and thus possibly nonzero $I B$ or $E B$ correlations, there should be no preference for a given handedness when averaging over many sources. More importantly, the existing measurements, which show that the offset $\alpha$ is small in the sources where it is measured, suggest that $I B$ and $E B$ correlations will be small.

If $\mathrm{CB}$ rotates the polarization by an angle $\alpha$, then part of the $E$ mode is rotated into a $B$ mode, $\delta \tilde{B}(\vec{l})=\tilde{E}(\vec{l}) \sin 2 \alpha \simeq$ $2 \alpha \tilde{E}(\vec{l})$, thus inducing nonzero $P_{l}^{I B}=2 \alpha P_{l}^{I E}$ and $P_{l}^{E B}=$ $2 \alpha P_{l}^{E E}$. A rotation-angle estimate is then obtained by comparing the measured $I B$ and $E B$ correlations with the measured $I E$ and $E E$ correlations, respectively.

A similar analysis can be done, alternatively and equivalently, using temperature-polarization two-point correlation functions $[18,20]$. This involves taking all pairs $\left(\vec{\theta}_{1}, \vec{\theta}_{2}\right)$ of points in the map, and then measuring correlations between the intensity $I$ and Stokes parameters $Q_{r}$ and $U_{r}$ measured in a coordinate system that is aligned with the line connecting the two points. Again, symmetry considerations suggest that, in the absence of $\mathrm{CB}$, $\left\langle I\left(\vec{\theta}_{1}\right) U_{r}\left(\vec{\theta}_{2}\right)\right\rangle=\left\langle Q_{r}\left(\vec{\theta}_{1}\right) U_{r}\left(\vec{\theta}_{2}\right)\right\rangle=0$. If the polarizations are rotated by an angle $\alpha$, then these parity-odd correlations are induced, with magnitudes $\left\langle I\left(\vec{\theta}_{1}\right) U_{r}\left(\vec{\theta}_{2}\right)\right\rangle=$ $2 \alpha\left\langle I\left(\vec{\theta}_{1}\right) Q_{r}\left(\vec{\theta}_{2}\right)\right\rangle$ and $\left\langle Q_{r}\left(\vec{\theta}_{1}\right) U_{r}\left(\vec{\theta}_{2}\right)\right\rangle=2 \alpha\left\langle Q_{r}\left(\vec{\theta}_{1}\right) Q_{r}\left(\vec{\theta}_{2}\right)\right\rangle$. The decision as to whether to use power spectra or correlation functions will depend on the noise properties of the map.

Discussion.-Here I have discussed measurements of a $\mathrm{CB}$ rotation of the linear polarization that varies as a function of position on the sky and derived rough upper limits to rotation-angle multipole moments and power spectra. I discussed how the analysis of future highresolution intensity-polarization images of high-redshift sources can be optimized with techniques analogous to those in CMB-polarization studies. 
The analysis presented here is meant primarily to be illustrative. The existing data are far from optimized for this particular measurement. First of all, I used only nine sources at redshifts $z \geq 2$, and the statistical weight is dominated by only half of those. Moreover, they are not uniformly spread on the sky (which is why the errors on the different $m$ moments for a given $l$ vary so widely), and this could give rise to pitfalls, perhaps weakening slightly the bounds to the lowest multipole moments.

Although a comparable sensitivity to a positiondependent rotation can, in principle, be obtained from existing CMB data, the analysis is difficult and has not yet been done. The simple exercise I have performed here is thus the strongest existing constraint on a positiondependent rotation, at least for a rotation that occurs at redshifts $0<z \lesssim 2$. A model that predicts rotation at $3<$ $z \lesssim 1100$ could still produce a signal in the CMB without violating the constraint I have derived. Likewise, a slightly stronger constraint can probably be obtained from the radio-galaxy data in Ref. [13], although for lower-redshift sources, and thus over a smaller baseline. Whether that constraint would be competitive with the one I have derived would, again, depend on the redshift dependence of $\alpha$ in any given model.

Improvements to the illustrative analysis I have done here should be straightforward. The error on the $\alpha_{l m}$ should scale simply as $N^{-1 / 2}$ with the number $N$ of sources, assuming similar image qualities to those obtained so far. Thus, for example, if the sample I used of $N \sim 4$ wellmeasured offsets can be improved to $N \sim 400$, the sensitivity will be competitive with that expected from Planck. However, progress can be accelerated, beyond $N^{-1 / 2}$, if more precise offset measurements can be obtained for at least some of these individual sources, either from better images, an improved analysis, or both. The goal of identifying the new physics responsible for cosmic acceleration will hopefully motivate such empirical investigations.

I thank S. di Serego Alighieri, R. Caldwell, V. Gluscevic, and A. Readhead for useful discussions. This work was supported by DOE Grant No. DE-FG03-92-ER40701, NASA Grant No. NNX10AD04G.
[1] E. J. Copeland, M. Sami, and S. Tsujikawa, Int. J. Mod. Phys. D 15, 1753 (2006); R. R. Caldwell and M. Kamionkowski, Annu. Rev. Nucl. Part. Sci. 59, 397 (2009); A. Silvestri and M. Trodden, Rep. Prog. Phys. 72, 096901 (2009); A. J. Albrecht et al., arXiv:astro-ph/ 0609591; E. V. Linder, Rep. Prog. Phys. 71, 056901 (2008); J. Frieman, M. Turner, and D. Huterer, Annu. Rev. Astron. Astrophys. 46, 385 (2008).

[2] B. Ratra and P. J.E. Peebles, Phys. Rev. D 37, 3406 (1988); C. Wetterich, Astron. Astrophys. 301, 321 (1995); K. Coble, S. Dodelson, and J. A. Frieman, Phys. Rev. D 55, 1851 (1997); M. S. Turner and M. J. White, Phys. Rev. D 56, R4439 (1997); R. R. Caldwell, R. Dave, and P. J. Steinhardt, Phys. Rev. Lett. 80, 1582 (1998).

[3] S. M. Carroll, Phys. Rev. Lett. 81, 3067 (1998).

[4] S. M. Carroll, G. B. Field, and R. Jackiw, Phys. Rev. D 41, 1231 (1990).

[5] A. Lue, L. M. Wang, and M. Kamionkowski, Phys. Rev. Lett. 83, 1506 (1999); N. F. Lepora, arXiv:gr-qc/9812077.

[6] B. Feng et al., Phys. Rev. Lett. 96, 221302 (2006); P. Cabella, P. Natoli, and J. Silk, Phys. Rev. D 76, 123014 (2007); T. Kahniashvili, R. Durrer, and Y. Maravin, Phys. Rev. D 78, 123009 (2008); J. Q. Xia et al., Astrophys. J. 679, L61 (2008); Astron. Astrophys. 483, 715 (2008); E. Y. Wu et al. (QUaD Collaboration), Phys. Rev. Lett. 102, 161302 (2009); L. Pagano et al., Phys. Rev. D 80, 043522 (2009); E. Komatsu et al., arXiv:1001.4538.

[7] M. Pospelov, A. Ritz, and C. Skordis, Phys. Rev. Lett. 103, 051302 (2009); M. Li and X. Zhang, Phys. Rev. D 78, 103516 (2008); R. R. Caldwell (unpublished).
[8] S. Gardner, Phys. Rev. Lett. 100, 041303 (2008).

[9] M. Kamionkowski, Phys. Rev. Lett. 102, 111302 (2009); V. Gluscevic, M. Kamionkowski, and A. Cooray, Phys. Rev. D 80, 023510 (2009); A. P. S. Yadav et al., Phys. Rev. D 79, 123009 (2009).

[10] B. Nodland and J. P. Ralston, Phys. Rev. Lett. 78, 3043 (1997).

[11] T. J. Loredo, E. E. Flanagan, and I. M. Wasserman, Phys. Rev. D 56, 7507 (1997); D. J. Eisenstein and E. F. Bunn, Phys. Rev. Lett. 79, 1957 (1997).

[12] J. F. C. Wardle, R. A. Perley, and M. H. Cohen, Phys. Rev. Lett. 79, 1801 (1997).

[13] J. P. Leahy, arXiv:astro-ph/9704285.

[14] S. M. Carroll and G. B. Field, Phys. Rev. Lett. 79, 2394 (1997).

[15] A. Cimatti, S. di Serego Alighieri, G. B. Field, and R. A. E. Fosbury, Astrophys. J. 422, 562 (1994).

[16] S.d. S. Alighieri, F. Finelli, and M. Galaverni, Astrophys. J. 715, 33 (2010).

[17] P. P. Kronberg, C. C. Dyer, and H.-J. Röser, Astrophys. J. 472, 115 (1996).

[18] M. Kamionkowski, A. Kosowsky, and A. Stebbins, Phys. Rev. D 55, 7368 (1997); Phys. Rev. Lett. 78, 2058 (1997); P. Cabella and M. Kamionkowski, arXiv:astro-ph/ 0403392.

[19] M. Zaldarriaga and U. Seljak, Phys. Rev. D 55, 1830 (1997); U. Seljak and M. Zaldarriaga, Phys. Rev. Lett. 78, 2054 (1997).

[20] A. Stebbins, arXiv:astro-ph/9609149; M. Kamionkowski et al., Mon. Not. R. Astron. Soc. 301, 1064 (1998). 